\title{
A Plasma Protein Indistinguishable from Ribosomal Protein S19
}

\author{
Conversion to a Monocyte Chemotactic Factor by a Factor \\ XIlla-Catalyzed Reaction on Activated Platelet Membrane \\ Phosphatidylserine in Association with Blood Coagulation
}

\author{
Umeko Semba, ${ }^{*}$ Jun Chen, ${ }^{*}$ Yoshihiko Ota, ${ }^{*}$ \\ Nan Jia, ${ }^{*}$ Hidetoshi Arima, ${ }^{\dagger}$ Hiroshi Nishiura, ${ }^{*}$ \\ and Tetsuro Yamamoto* \\ From the Departments of Molecular Pathology* and Physical \\ Pharmaceutics, ${ }^{\dagger}$ Faculty of Life Sciences, Kumamoto University \\ Graduate School, Kumamoto, Japan
}

\begin{abstract}
A monocyte-chemoattracting factor is generated during blood coagulation and during clotting of platelet-rich plasma. This chemotactic factor attracts monocytes as a ligand of the $\mathrm{C} 5 \mathrm{a}$ receptor; however, it inhibits $\mathrm{C} 5 \mathrm{a}$-induced neutrophil chemotaxis as an apparent receptor antagonist. The curious dual function of the serum monocyte chemotactic factor resembles that of the cross-linked homodimer of ribosomal protein S19 (RP S19). Indeed, the inactive precursor of the monocyte chemotactic factor was present in plasma, and the precursor molecule and RP S19, as well as the active form and the RP S19 dimer, were indistinguishable in terms of immunological reactivity and molecular size. Coagulation factor XIIIa, plasma transglutaminase, and membrane phosphatidylserine on the activated platelets were required for conversion of the precursor to the active form. In addition, the precursor molecule in plasma could be replaced by wild-type recombinant RP S19 but not by mutant forms of it. These results indicate that a molecule indistinguishable from RP S19 was present in plasma, and that the RP S19-like molecule was converted to the active form by a transglutaminase-catalyzed reaction on a scaffold that included the phosphatidylserine-exposed platelet membrane. (Am J Pathol 2010, 176:1542-1551; DOI: 10.2353/ajpath.2010.090720)
\end{abstract}

Studies by our group have been ongoing with regard to the cross-linked homodimer of ribosomal protein S19 (RP S19) as a monocyte-selective chemoattractant factor. ${ }^{1,2}$ The chemotactic function of the RP S19 dimer is a typical extraribosomal activity and to gain this activity the transglutaminase-catalyzed intermolecular cross-linkage between GIn137 and Lys122 is needed. ${ }^{3}$ The RP S19 dimer is formed in apoptosis-initiated cells and then extracellularly released. ${ }^{4-6}$ The RP S19 dimer has so far been isolated from rheumatoid arthritis synovial lesions and from atherosclerotic lesions of the aorta. ${ }^{7,8}$ The RP S19 dimer induces monocyte chemoattraction as an agonistic ligand of the C5a receptor; however, this dimer inhibits neutrophil chemoattraction induced by $\mathrm{C} 5 \mathrm{a}$, the complement C5-derived pan-leukocyte chemotactic molecule, as an apparent antagonist of the C5a receptor. ${ }^{9-11}$

More than 20 years ago, we reported a novel monocyte-selective chemoattracting factor that was present in serum but not in plasma. The monocyte chemotactic factor was generated during blood coagulation via a mechanism dependent on the enzymatic activity of factor XIIIa, the plasma transglutaminase. The chemotactic factor was distinguished from C5a by its monocyte selectivity and by its large molecular size. Despite these differences, we thought at that time that the origin of the chemotactic factor was also complement C5 because the chemotactic factor was adsorbed by anti-C5 antibody

Supported by a grant-in aid for Scientific Research (C) (KAKENHI 21590441 to T.Y.) from the Ministry of Education, Culture, Sports, Science, and Technology, Japan.

Accepted for publication November 10, 2009.

Address reprint requests to Tetsuro Yamamoto, M.D., Department of Molecular Pathology, Faculty of Life, Kumamoto University, 2-2-1 Honjo, Kumamoto 860-0811, Japan. E-mail: tetsu@gpo.kumamoto-u.ac.jp. 
beads and because the chemoattraction was inhibited by a C5a receptor antagonist. ${ }^{12,13}$

However, we noticed later that the RPS19 dimer, but not the monomer, possesses antigen epitopes recognized by anti-C5a monoclonal antibodies. ${ }^{9}$ This raised the possibility that the monocyte chemotactic factor in serum could be the RP S19 dimer. If this were the case, one big question was whether the precursor, RP S19, is present in normal plasma.

In the current study, we first re-examined the serum monocyte chemotactic factor in light of the recent findings on the functions of the RP S19 dimer using anti-RP S19 antibodies. We then studied its precursor molecule in plasma and the conversion mechanism to the active form in association with blood coagulation. In these studies, we revealed both the presence of a molecule in plasma indistinguishable from RP S19 and the mechanism to convert this molecule to the monocyte chemotactic factor. We report here that the active form of factor XIII and thrombin-activated platelets are involved as the enzymatic catalyst and the reaction scaffold, respectively, in the activation mechanism.

\section{Materials and Methods}

\section{Reagents and Others}

RPMI 1640 medium and HBSS were purchased from Nissui Pharmaceutical (Tokyo, Japan). Fetal bovine serum was a product of Invitrogen Life Technologies (Paisley, Scotland). Ficoll-Paque Plus and ECL Plus Western blotting detection system were obtained from Amersham Biosciences KK (Tokyo, Japan). Bovine serum albumin, biotin N-hydroxysuccinimide ester and p-chloromercuriphenylsulfonic acid (PCMPS) were obtained from Sigma-Aldrich (St. Louis, MO). A multiwell chamber for chemotaxis assay was a product of Neuro Probe (Bethesda, MD). Nucleopore filters were purchased from Nucleopore (Pleasant, CA). Congenital deficiency of blood coagulation factor XIII plasma was obtained from George King Bio-Medical (Overland Park, KS). Purified human blood coagulation factor XIII was a gift from the Institute of Chemo Sero Therapy (Kumamoto, Japan). Polyvinylidene fluoride membrane was purchased from Millipore (Bedford, MA). Block Ace was purchased from DS Phama Biomedical (Osaka, Japan). All other chemicals were obtained from Nacalai Tesque (Kyoto, Japan) or from Wako Pure Chemicals (Osaka, Japan) unless otherwise specified.

Carinactivase-1 (CA-1), which is a prothrombin-activating metalloprotease obtained from venom of snake (Echis carinatus) ${ }^{14}$ was a gift from Prof. T. Morita of Meiji College of Pharmacy (Tokyo, Japan). The C5a receptor antagonistic peptide, NMePhe-Lys-Pro-dCha-dCha-dArg, was synthesized as described previously. ${ }^{15}$ Anti-RP S19 protein rabbit IgG and rabbit IgG against a synthetic C-terminal peptide of RP S19 (from Ala135 to His145 with a Cys at the C-terminal) (Ala-Gly-Gln-Val-Ala-Ala-Ala-Asn-Lys-Lys-His-Cys) were prepared as described previously. ${ }^{6}$ IgG fractions of these antisera were prepared using a HiTrap Protein G HP column (Amersham Biosciences KK), according to the manufactur- er's instruction. Aliquots of the anti-RP S19 peptide IgG as well as normal rabbit lgG were conjugated with biotin using biotin $N$-hydroxysuccinimide ester according to the instruction manual prepared by the manufacturer. For preparation of immunobeads, an aliquot of the anti-RP S19 protein rabbit IgG was conjugated to Cyanogen Bromide-activated Sepharose 4 Fast Flow (GE Health care BioScience, Piscataway, NJ) at $\mathrm{pH} 8.5$, according to the manufacturer's instruction.

A recombinant $\mathrm{C5a}$, a recombinant wild-type RP S19, a recombinant Gln137Asn mutant of RP S19 (Gln137Asn-RP S19), and a recombinant Lys23, 24, 27, 29Ala mutant of RP S19 (Lys23, 24, 27, 29Ala-RP S19) were prepared using an Escherichia coli expression system with pET32a vector and Rosseta gami(B) Lys-S as the host bacteria as described previously. ${ }^{11,16}$

\section{Preparation of Liposomes}

The lipids were dissolved in chloroform in 10-ml roundbottom flasks, and a film was obtained after evaporation of the solvent under vacuum at $20^{\circ} \mathrm{C}$ overnight using a Heidolph rotating desiccator. The dried film was then hydrated for 24 hours with sterile, deionized water at $4^{\circ} \mathrm{C}$ to produce large multilamellar vesicles. The suspension was finally sonicated at $4^{\circ} \mathrm{C}$ for 2 minutes $(115 \mathrm{~V}, 80 \mathrm{~W}, 60 \mathrm{~Hz})$ with a G112SP1G model sonicator (Laboratory Supplies, Hicksville, NY) to obtain small unilamellar liposomes. Three different kinds of liposomes were prepared in terms of their phosphatidylserine contents: 0, 10, and 30\% phospholipid. The average size of the liposomes was $0.4 \mu \mathrm{m}$ in diameter. The opalescent liposome preparation was stored up to 1 month at $4^{\circ} \mathrm{C}$. When we substituted the platelets with the liposomes, we mixed one of the liposome preparations into platelet-poor plasma at a final concentration of $78 \mu \mathrm{g} / \mathrm{ml}$.

\section{Preparation of Blood-Derived Serum, Platelet-Rich Plasma, Platelet-Poor Plasma, Plasma-Derived Sera, and Washed Platelets}

To prepare blood-derived serum, blood was taken from the peripheral veins of healthy individuals without any reagent and coagulated in a glass container for 30 minutes at $22^{\circ} \mathrm{C}$. To prepare plasma, peripheral venous blood was taken in the presence of a $1 / 9$ volume of $3.2 \%$ citrate- $3 \mathrm{Na}-2 \mathrm{H}_{2} \mathrm{O}$, and the anti-coagulated blood was centrifuged at $140 \times g$ for 15 minutes at $22^{\circ} \mathrm{C}$. The supernatant was centrifuged again at $140 \times g$ for 5 minutes at $22^{\circ} \mathrm{C}$ to obtain platelet-rich plasma. The platelet-rich plasma was centrifuged at $7000 \times g$ for 10 minutes at $22^{\circ} \mathrm{C}$. The supernatant was used as platelet-poor plasma, and the pellet was washed with PBS to prepare washed platelets. Aliquots $(1 \mathrm{ml})$ of the platelet-rich plasma and the platelet-poor plasma were coagulated by adding CA-1 (1 $\mu \mathrm{g}$ in $2.3 \mu \mathrm{l}$ ) and $500 \mathrm{mmol} / \mathrm{L} \mathrm{CaCl} \mathrm{Cl}_{2}$ (33 $\mu l)$ for 30,60 , or 90 minutes at $37^{\circ} \mathrm{C}$, and the coagula were centrifuged at $10,000 \times g$ for 10 minutes at $22^{\circ} \mathrm{C}$ to obtain the supernatants as platelet-rich plasma-derived serum and platelet-poor plasma-derived serum. When the sera were subjected to the chemotaxis assay, they 
were heat-treated for 30 minutes at $56^{\circ} \mathrm{C}$ to deactivate the complement system, and the aggregated proteins were discarded by centrifugation at $10,000 \times g$ for 10 minutes at $22^{\circ} \mathrm{C}$. This process was required to prevent complement activation during the chemotaxis chamber assay for 90 minutes at $37^{\circ} \mathrm{C}$.

In some experiments, the platelet-rich plasma was supplemented with $\mathrm{p}$-chloromercuriphenylsulfonic acid, glucosamine, $\mathrm{N}$-acetylglucosamine or annexin $\mathrm{V}$, and then the platelet-rich plasma-derived serum was prepared.

\section{Immunodepletion of an RP S19-Like Molecule in Plasma with Antibody Beads Before Preparation of Platelet-Rich Plasma-Derived Serum}

Platelet-rich plasma was prepared as above and divided into three aliquots. In these aliquots, the platelet-poor plasma and platelets were separated by centrifugation. The platelets were kept on ice. The platelet-poor plasma part was mixed with a 1/10 volume of either the anti-RP S19 rabbit IgG beads, normal rabbit IgG beads, or PBS and then incubated for 30 minutes at $37^{\circ} \mathrm{C}$ and centrifuged at $10,000 \times g$ for 5 minutes at $4^{\circ} \mathrm{C}$. The supernatant was recovered and mixed again with a $1 / 10$ volume of the new beads or PBS to repeat the process. The immunodepleted plasma was mixed with the platelets to reconstitute the platelet-rich plasma, and the platelet-rich plasma-derived serum was then prepared by the 60-minute incubation with CA-1. After deactivation of the complement system via heat treatment, the samples were subjected to the chemotaxis assay. In some experiments, plasma immunodepleted for the RP S19-like molecule was reconstituted with either the wild-type recombinant RP S19 or one of the mutants of RP S19 (Gln137Asn-RP S19 and Lys23, 24, 27, 29Ala-RP S19), and the platelet-rich plasma-derived sera were subjected to the chemotaxis assay.

\section{Preparation of Platelet-Rich Plasma-Derived Serum Using Factor XIII-Deficient Plasma}

Two aliquots of the factor XIII-deficient plasma (400 $\mu$ l) were prepared. Separately, washed platelets were prepared from the same volume as the aliquots of platelet-rich plasma of a healthy individual. One aliquot of the deficient plasma was reconstituted with a small volume $(3 \mu \mathrm{l})$ of a purified factor XIII concentrate $(130 \mathrm{U} / \mathrm{ml})$ to the normal factor XIII concentration $(1 \mathrm{U} / \mathrm{ml})$, and the other was mixed with PBS at the same volume. These aliquots were then mixed with the washed platelets to reconstitute platelet-rich plasma, and the platelet-rich plasma-derived serum was then prepared by the 60-minute incubation with CA-1. After deactivation of the complement system by the heat treatment, the samples were subjected to the chemotaxis assay.

\section{Chemotaxis Assay}

Mononuclear cells and neutrophils were isolated from heparinized human venous blood of healthy donors ac- cording to the method of Fernandez et $\mathrm{al}^{17}$ as described previously. ${ }^{18}$ The mononuclear cell fraction contained monocytes at $\sim 20 \%$, and almost all of the cells in the neutrophil fraction were neutrophils themselves. The mononuclear cells and neutrophils were, respectively, suspended at a cell density of $1 \times 10^{6}$ cells $/ \mathrm{ml}$ in RPMI 1640 containing 10\% fetal bovine serum and at a cell density of $2 \times 10^{6}$ cells $/ \mathrm{ml}$ in HBSS containing $0.5 \%$ bovine serum albumin for the multiwell chamber assay.

The multiwell chamber assay was performed according to the method of Falk et al $^{19}$ using a Nucleopore filter with a pore size of $5 \mu \mathrm{m}$ for monocytes and of $3 \mu \mathrm{m}$ for neutrophils as described previously. ${ }^{18}$ After incubation for 90 minutes, each membrane was separated, fixed with methanol, and stained with Giemsa solution. The total number of monocytes or of neutrophils migrated beyond the lower surface of the membrane was counted in five microscopic high-power fields. The results are expressed as the number of migrated monocytes or neutrophils. Each experiment was performed in triplicate.

\section{Polyacrylamide Gel Electrophoresis in Presence of SDS and Western Blotting}

For Western blotting analysis, the plasma and serum samples were pretreated with dextran sulfate and with acetone, in this order, to remove extra-large molecules and small ions in the samples; otherwise, the pattern of electrophoresis became irregular. Initially, dextran sulfate sodium were added at a final concentration of $2 \%$ to the samples and centrifuged at $35,000 \times g$ for 30 minutes at $4^{\circ} \mathrm{C}$ according to the method of Cornwell and Kruger. ${ }^{20}$ The supernatants were recovered and added with four times volume of acetone precooled at $-20^{\circ} \mathrm{C}$ and vigorously mixed with a vortex mixer. The mixtures were stood for 60 minutes at $-20^{\circ} \mathrm{C}$, and centrifuged at $15,000 \times g$ for 10 minutes at $4^{\circ} \mathrm{C}$. The pellets were recovered, dissolved into the electrophoresis sample buffer containing SDS, $\beta$-mercaptoethanol, and urea, heat treated for 20 minutes at $42^{\circ} \mathrm{C}$, and subjected to SDS-polyacrylamide gel electrophoresis and Western blotting analyses.

SDS-polyacrylamide gel electrophoresis was performed with $12.5 \%$ acrylamide. The polyacrylamide gels were subjected to transfer the proteins to polyvinylidene fluoride membrane using a semidry electrophoretic transfer cell, Trans-Blot SD (Bio-Rad, Richmond, CA). After washing twice in PBS, the membrane was treated with $0.4 \%$ Block Ace for 30 minutes at $22^{\circ} \mathrm{C}$, then reacted with the biotinylated anti-RP S19 peptide rabbit IgG (final concentration 1 $\mu \mathrm{g} / \mathrm{ml}$ ) at $4^{\circ} \mathrm{C}$ overnight, followed by incubation with a 500fold diluted streptavidin-biotin-peroxidase complex solution for 20 minutes at $22^{\circ} \mathrm{C}$. The peroxidase activity on the membrane was converted to an enzyme reaction product using ECL Plus western blotting detection system and the product was quantified using an image capturing unit, LAS4000mini (Fuji Film, Tokyo, Japan) with an analysis software, Multi Gauge (Fuji Film). 


\section{Results}

In this study, we repeated each experiment and show a representative result after confirming reproducibility.

\section{Generation of Monocyte Chemotactic Factor Concomitant with Blood Coagulation or with Platelet-Rich Plasma Clotting}

As we previously reported, serum but not plasma possesses monocyte-selective chemotactic capacity. ${ }^{12,13}$ To examine the characteristics of the monocyte chemotactic factor and its mechanism of generation during blood coagulation, we prepared platelet-poor plasma-derived serum and platelet-rich plasma-derived serum and measured the chemotactic capacities of the plasma-derived sera and blood-derived serum. In the usual coagulation cascade, the rate of thrombin generation from prothrombin is very much affected by the presence of activated platelets on which the protease cascade reaction can take place. To generate thrombin at a nearly equal amount and at a rapid rate regardless of the presence or absence of platelets, we used CA-1 as a direct prothrombin activator to prepare the plasma-derived sera in our current experiments.

We prepared platelet-rich plasma and platelet-poor plasma from blood of normal individuals. We induced plasma clotting by adding CA-1 to the platelet-rich plasma or to the platelet-poor plasma for various time periods (30, 60 , and 90 minutes) and separated the plasma-derived sera by centrifugation. Meanwhile, we prepared serum from the same individual's blood that was drawn without anticoagulant. After heat treatment for 30 minutes at $56^{\circ} \mathrm{C}$ to deactivate the complement system, we measured the monocyte chemotactic capacity in the plasma-derived sera as well as in the blood-derived serum. The results are shown in Figure 1. The monocyte chemotactic activity emerged in the serum derived from platelet-rich plasma, depending on the incubation period. A rapid increase in chemotactic capacity was observed until 60 minutes, and the activity gradually increased further until 90 minutes. The monocyte chemotactic capacities of the 60- and 90-minute samples were comparable with that of the blood-derived serum. In this comparison, the monocyte chemotactic capacity generated by the platelet-poor plasma-derived serum was significantly lower.

\section{Immunological and Functional Indistinguishability Between Monocyte Chemotactic Factor in Serum and RP S19 Dimer}

To examine the functional identities of the monocyte chemotactic factors generated in platelet-rich plasma-derived serum and in blood-derived serum, we measured their chemotactic capacities to attract neutrophils. Neither of them attracted neutrophils (data not shown). In terms of monocyte selectivity, the chemoattracting factor in the sera and the RP S19 dimer were similar. As described above, the RP S19 dimer attracts monocytes as an agonistic ligand of the C5a receptor and does not attract neutrophils; furthermore,

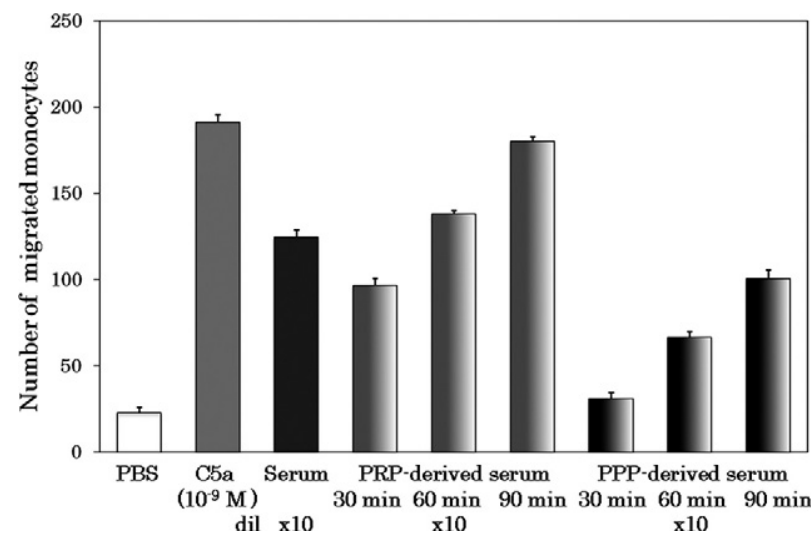

Figure 1. Time-dependent generation of monocyte-attracting factor in plateletrich plasma concomitant with coagulation. Platelet-rich plasma (PRP; $1 \mathrm{ml}$ ) and platelet-poor plasma (PPP; $1 \mathrm{ml}$ ) were coagulated for 30, 60, or 90 minutes at $37^{\circ} \mathrm{C}$ by adding CA-1 $(1 \mu \mathrm{g}$ in $2.3 \mu \mathrm{l})$ and $500 \mathrm{mmol} / \mathrm{L} \mathrm{CaCl}_{2}(33 \mu \mathrm{l})$. After centrifugation at $10,000 \times g$ for 10 minutes at $22^{\circ} \mathrm{C}$, the supernatants were recovered as PRP-derived serum (gradient light gray bars) and PPP-derived serum (gradient dark gray bars), respectively. Blood-derived serum (black bar) was prepared from spontaneously coagulated blood in the usual way. These sera were heat treated for 30 minutes at $56^{\circ} \mathrm{C}$ to deactivate the complement system and centrifuged at $10,000 \times g$ for 10 minutes at $22^{\circ} \mathrm{C}$. The supernatants were subjected to the monocyte chemotaxis assay in a multiwell chamber. As negative and positive controls in the chemotaxis assay, PBS (white bars) and $10^{-9} \mathrm{M}$ C5a (grey bars) were used, respectively. The chemotactic capacity is shown as the monocyte number counted in five microscopic high-power fields. Each experiment was performed in triplicate, and the column height indicates the mean value with SD bar. dil, fold dilution.

it suppresses the neutrophil migration induced by $\mathrm{C5a}$ as an apparent antagonist of the C5a receptor. ${ }^{9}$ Therefore, we examined the monocyte chemotactic factor in the sera for a dual function at the leukocyte $\mathrm{C5a}$ receptor. The monocyte chemoattractions induced by both of the sera or by C5a were almost equally inhibited by a C5a receptor antagonist peptide, NMePhe-Lys-Pro-dCha-dCha-dArg, at $10^{-6}$ $\mathrm{M}$ (data not shown). The sensitivity to the antagonist was equivalent to that of the RP S19 dimer, as reported previously. ${ }^{9}$ We then examined the effect of sera on the neutrophil C5a receptor. A multiwell chamber assay for neutrophils does not work when the lower chamber is filled with a solution containing very high doses of proteins such as neat plasma or neat serum (the mechanism is unknown). We therefore used 10-, 100-, and 1000-fold diluted plasma or sera in the neutrophil chemotaxis inhibition assay. As shown in Figure 2, both of the sera inhibited the C5a-induced neutrophil chemotaxis in the same as previously reported for the RP S19 dimer. $^{9}$ In contrast, neither the plasma nor the platelet-poor plasmaderived serum exhibited the inhibitory capacity.

In terms of functional characteristics, the monocyte chemotactic factor in the sera and the RP S19 dimer were indistinguishable. We then examined the immunological similarity of the serum factor to RP S19. In this experiment, we twice treated the platelet-rich plasma-derived serum in batch-wise with anti-RP S19 IgG-beads and measured the remaining monocyte chemotactic capacity. The reason for repeating the immunoabsorption was to compensate for a low efficacy of the batchwise method. As shown in Figure 3, the monocyte chemotactic activity of the serum was adsorbed out by the anti-RP S19 IgG beads but not by the normal rabbit IgG beads. The monocyte chemotactic factor 


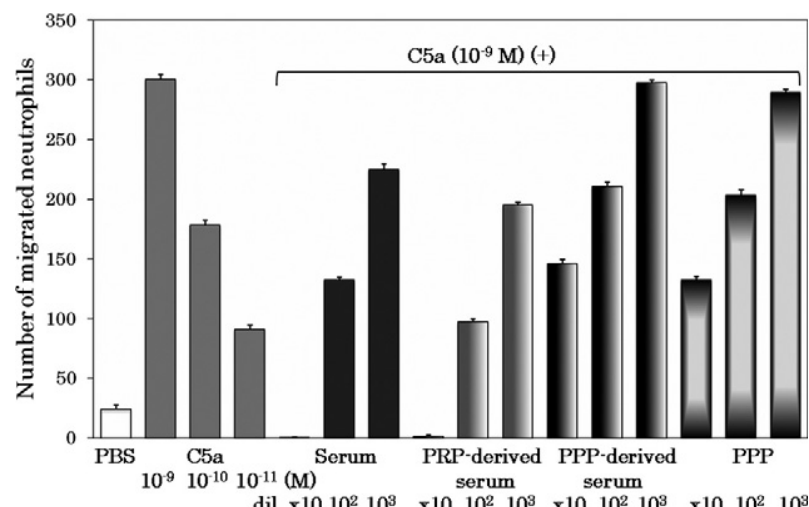

Figure 2. Inhibition of $\mathrm{C} 5$ a-induced neutrophil chemotaxis by blood-derived serum or by platelet-rich plasma-derived serum. To examine whether the bloodderived serum and the platelet-rich plasma-derived serum possessed the antagonist capacity to the $\mathrm{C} 5 \mathrm{a}$ receptor of neutrophils, the neutrophil chemotaxis assay with $10^{-9} \mathrm{M}$ C5a was performed in the presence of three different concentrations of these sera from 10-fold dilution to 1000-fold dilution. The concentration-activity relationship of $\mathrm{C} 5 \mathrm{a}$ is shown (grey bars). The platelet-poor plasma and platelet-poor plasma-derived serum were used as negative controls.

in the blood-derived serum was adsorbed by the anti-RP S19 lgG-beads at the same extent as the platelet-rich plasma-derived serum (data not shown).

These results indicate that the monocyte chemotactic factor in platelet-rich plasma-derived serum and that in blood-derived serum are identical, and that the serum monocyte chemotactic factor and the RP S19 dimer are immunologically and functionally indistinguishable.

\section{RP S19-Like Molecule in Plasma as Precursor of Monocyte Chemotactic Factor in Platelet-Rich Plasma-Derived Serum}

Platelets are now thought to synthesize proteins on activation using RNA transcripts and protein synthetic machinery, including ribosomes, provided by parent megakaryocytes. ${ }^{21,22}$ Hence, there was a possibility that the precursor of the monocyte chemotactic factor in the

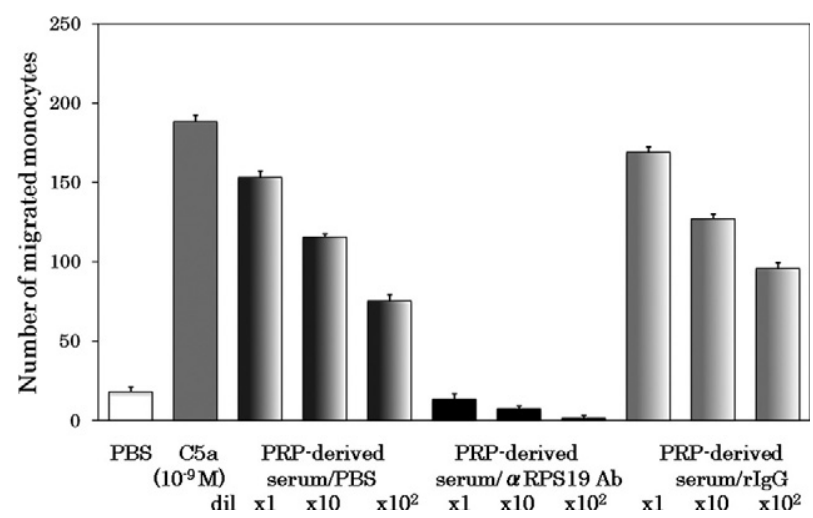

Figure 3. Absorption of monocyte-attracting factor in platelet-rich plasmaderived serum by anti-RP S19 antibody beads. The complement-deactivated platelet-rich plasma-derived serum was treated with either anti-RP S19 rabbit IgG-beads (PRP-derived serum/ $\alpha$ RPS19 Ab) or with normal rabbit IgG-beads (PRP-derived serum/rIgG) in batchwise twice, and the monocyte chemotactic capacity remained was measured at three serial log-scale dilutions. As a negative control, the serum was diluted with PBS at the same volume as the beads (PRP-derived serum/PBS). Positive and negative controls for the chemotaxis assay were $10^{-9} \mathrm{M}$ C5a and PBS, respectively.

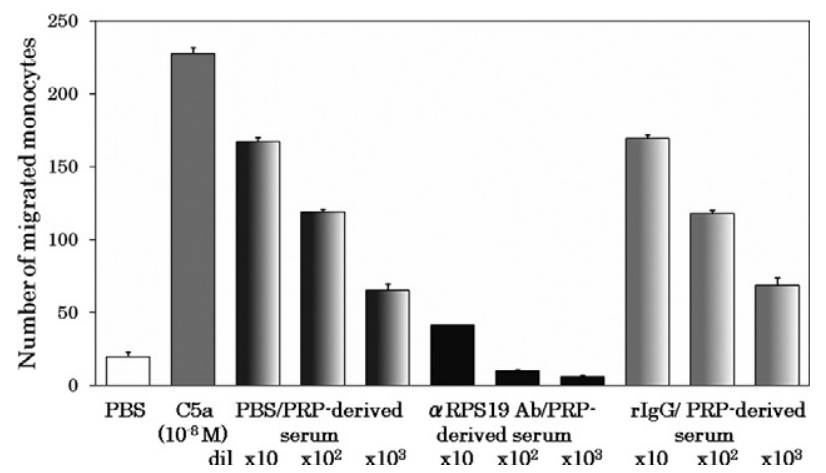

Figure 4. Depletion of the precursor of the monocyte-attracting factor in plasma with anti-RP S19 antibody beads. As described in Materials and Methods, aliquots of platelet-poor plasma (PPP) were pretreated with PBS (PBS/), with the anti-RP S19 IgG-beads ( $\alpha$ RPS19 Ab/), or with the normal rabbit IgG beads ( $\mathrm{rIgG} /$ ), and were added with platelets to reconstitute PRP. The reconstituted PRP samples were coagulated with CA-1, and the sera of them (PRP-derived serum) were prepared. After the complement deactivation, the samples were subjected to the monocyte chemotaxis assay at three serial log-scale dilutions. Positive and negative controls for the chemotaxis assay were $10^{-8} \mathrm{M} \mathrm{C5} \mathrm{a}$ and $\mathrm{PBS}$, respectively.

platelet-rich plasma-derived serum was RP S19 derived from the platelet ribosomes or newly synthesized by platelets on thrombin activation. Otherwise, the RP S19like molecule should be present in plasma. To examine these possibilities, we initially treated platelet-poor plasma either with the anti-RP S19 IgG beads or with the normal rabbit IgG beads and then reconstituted the platelet-rich plasma by adding back the washed platelets. We repeated the batchwise immunoadsorption process with the platelet-poor plasma twice to increase the efficacy of the immunodepletion. We finally prepared the plateletrich plasma-derived sera and measured the monocyte chemotactic capacity generated by them. The results are shown in Figure 4. The generation of monocyte chemotactic activity in the platelet-rich plasma-derived serum was made negligible by the preimmunoadsorption of plasma with anti-RP S19 antibody beads. These results indicated that the monocyte chemotactic factor generated during coagulation of platelet-rich plasma did not derive from the platelets but was present in plasma.

\section{Western Blotting Analyses of Plasma and Platelet-Rich Plasma-Derived Serum with Anti-RP S19 Antibodies}

We then tried to detect the RP S19-like molecule in plasma and in plasma-derived serum by means of Western blotting with the anti-RP S19 rabbit IgG. Neither naked plasma nor serum was suitable for Western blotting because of the high concentration of proteins and because of the high concentration of small organic molecules and ions. We therefore removed both of them from plasma and serum and subjected what was left to the analyses. As shown in Figure 5, one and two immune reactive bands are respectively observed in plasma and serum. The electrophoretic mobility of the reactive band in plasma and the band in serum corresponded to RP S19 and the RP S19 homodimer. Because the sample denaturing buffer contained a reducing reagent, the mol- 


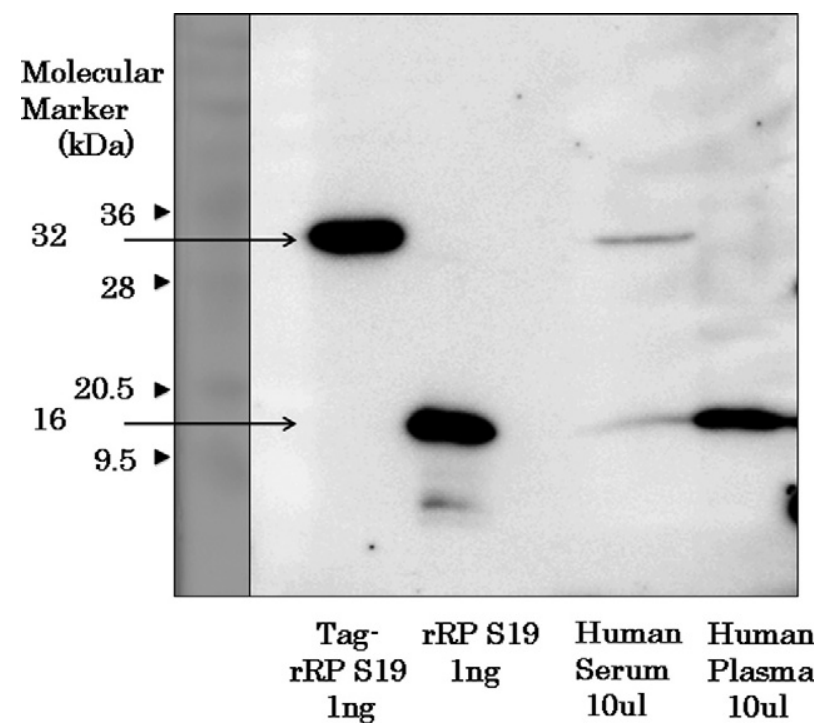

Figure 5. Demonstration by western blotting of the presence of RP S19-like molecules in plasma and its possible dimerization during blood coagulation. After removal of extra-large molecules and small organic molecules and ions, plasma and serum samples were treated with SDS, urea, and $\beta$-mercaptoethanol and subjected to polyacrylamide gel electrophoresis with $12.5 \%$ acrylamide in the presence of SDS. Recombinant RP S19 proteins with molecular tags (Tag-rRP S19) or without the tags (rRP S19) were used as positive controls. The Tag-rRP S19 possesses a molecular size $(32,000)$ similar to that of the RP S19 dimer. The molecules in the polyacrylamide gel were electrophoretically transferred to a polyvinylidene fluoride membrane. After blocking, the membrane was reacted with the biotinylated anti-RP S19 peptide rabbit IgG (final concentration, $1 \mu \mathrm{g} / \mathrm{ml}$ ) and then with streptavidinbiotin-peroxidase complex. The peroxidase activity on the membrane was visualized using an electrochemical luminescent method. The first lane is for molecular markers of which the molecular weights are shown at the left side as the number with arrowhead.

ecule with RP S19 dimer size should not be formed by an intermolecular disulfide bond. These results indicated that the RP S19-like molecule in plasma and the monocyte chemotactic factor were indistinguishable from RP S19 and from the RP S19 homodimer, respectively, not only in their immunological reactivity but also in their molecular sizes.

\section{Involvement of Factor XIIla into}

Monocyte-Attracting Factor Generation in Association with Clotting of Platelet-Rich

\section{Plasma}

Our previous study strongly suggested that a transglutaminase-catalyzed reaction played an essential role in the generation of monocyte chemotactic RP S19 dimer in the apoptosis-initiated cells., ${ }^{4,5}$ In our experimental settings in vitro, not only intracellular type 2 transglutaminase but also the plasma transglutaminase, factor XIIla, crosslinked RP S19 intermolecularly and generated the monocyte chemotactic RP S19 dimer. ${ }^{3,5}$ We therefore assumed the participation of factor XIII in plasma or the factor XIII A subunit in platelets in the generation of monocyte chemotactic factor in platelet-rich plasma as a zymogen of transglutaminase. To examine the contribution of factor XIII, we used a factor XIII-congenitallydeficient plasma to reconstitute the platelet-rich plasma

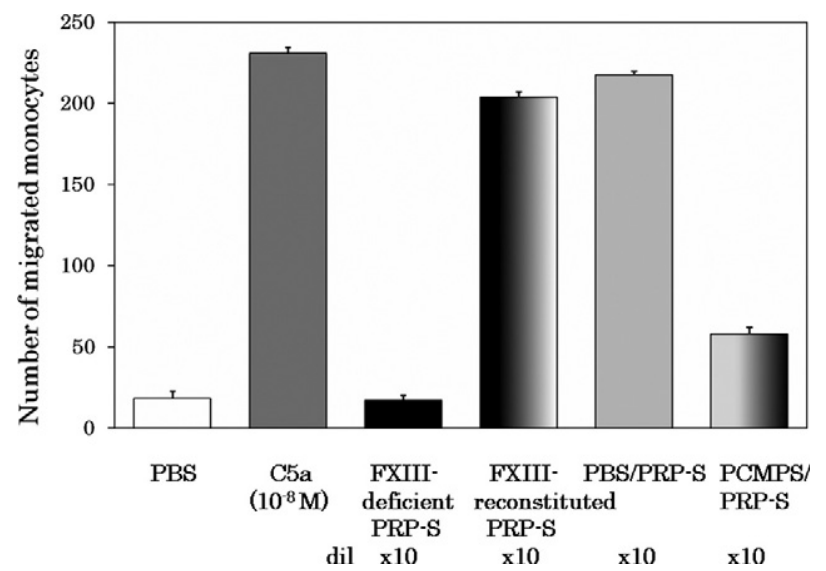

Figure 6. Involvement of factor XIIIa in monocyte-attracting factor generation during coagulation of platelet-rich plasma. Factor XIII-deficient plasma $(400 \mu \mathrm{l})$ was mixed with PBS $(3.1 \mu \mathrm{l})$ or with a factor XIII solution in PBS (130 $\mathrm{U} / \mathrm{ml}, 3.1 \mu \mathrm{l})$ at the final concentration of $1 \mathrm{U} / \mathrm{ml}$. The factor XIII-deficient plasma (FXIII-deficient) and the reconstituted plasma (FXIII-reconstituted) were respectively added with washed platelets prepared from $400 \mu \mathrm{l}$ of platelet-rich plasma to reconstitute the platelet-rich plasmas. Separately, 380 $\mu \mathrm{l}$ aliquots of normal platelet-rich plasma were added with $20 \mu \mathrm{l}$ of PBS (PBS/) or with $20 \mu \mathrm{l}$ of $20 \mathrm{mmol} / \mathrm{L}$ p-chloromercuriphenylsulfonic acid (PCMPS/) in PBS (final concentration, $1 \mathrm{mmol} / \mathrm{L}$ ). These platelet-rich plasmas were then coagulated for 60 minutes at $37^{\circ} \mathrm{C}$ by adding $500 \mathrm{mmol} / \mathrm{L} \mathrm{CaCl}_{2}$ $(13.2 \mu \mathrm{l})$ and CA-1 $(0.4 \mu \mathrm{g}, 0.93 \mu \mathrm{l})$. After centrifugation at $10,000 \times \mathrm{g}$ for 10 minutes at $22^{\circ} \mathrm{C}$, the supernatants, which are sera, were heat treated for 30 minutes at $56^{\circ} \mathrm{C}$, and further centrifuged at $10,000 \times g$ for 10 minutes at $22^{\circ} \mathrm{C}$. The supernatants (PRP-S, PRP-derived serum) were diluted 10-fold with PBS and subjected for the monocyte chemotaxis assay. Positive and negative controls for the chemotaxis assay were $10^{-8} \mathrm{M} \mathrm{C5} \mathrm{a}$ and PBS, respectively.

by adding washed normal platelets. As a positive control, the same deficient plasma reconstituted with purified factor XIII at a final concentration of $1 \mathrm{U} / \mathrm{ml}$ was used. As shown in Figure 6, only a negligible monocyte chemotactic capacity was generated in the factor XIII-deficient plasma even in the presence of normal platelets. The failure of chemotactic factor generation was completely compensated for by reconstitution with purified factor XIII at the normal concentration.

To examine whether the catalytic activity of factor XIIla as a transglutaminase was essential for monocyte-attracting factor generation, we performed the experiment with normal platelet-rich plasma under the simultaneous presence of PCMPS at a final concentration of $1 \mathrm{mmol} / \mathrm{L}$. Among the blood coagulation enzymes, only factor XIIla is a thiol enzyme sensitive to PCMPS. As shown in Figure 6 , the generation of monocyte chemotactic factor was strongly inhibited by the presence of PCMPS.

\section{Involvement of Phosphatidylserine Exposed on Activated Platelet Plasma Membrane in Monocyte Chemotactic Factor Generation in Association with Platelet-Rich Plasma Clotting}

We next addressed ourselves to the role of platelets in the generation of the monocyte chemotactic factor. The negatively charged membrane phospholipid, phosphatidylserine, is exposed on platelets on the platelet activation by the function of phospholipid scramblases. ${ }^{23}$ We assumed that membrane phosphatidylserines on the activated platelets would work as the negatively 


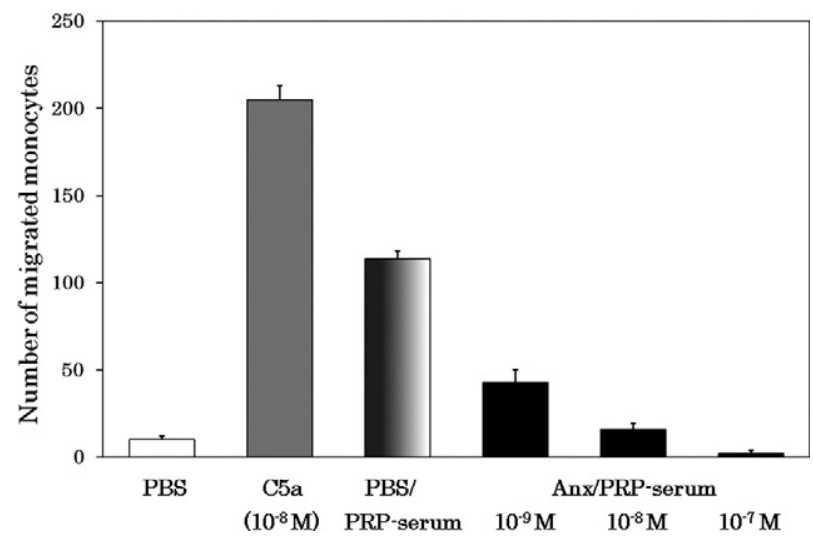

Figure 7. Inhibition by phosphatidylserine-binding annexin $\mathrm{V}$ of the generation of monocyte-attracting factor during coagulation in platelet-rich plasma. Aliquots of PRP were coagulated with CA-1 under the presence of $\mathrm{PBS}$ (PBS/) or of annexin V (Anx/) at $10^{-9}, 10^{-8}$, or $10^{-7} \mathrm{M}$, and sera of these aliquots (PRP serum) were subjected to the monocyte chemotaxis assay. Positive and negative controls for the chemotaxis assay were $10^{-8} \mathrm{M} \mathrm{C} 5 \mathrm{a}$ and PBS, respectively.

charged scaffold in the transglutaminase-catalyzed RP S19 cross-linkage as did heparin. ${ }^{3}$ Separately, there is a report that the recognition of phosphatidylserine moieties on aged neutrophils by macrophages was inhibited by 10 $\mathrm{mmol} / \mathrm{L}$ glucosamine but not by $\mathrm{N}$-acetylglucosamine. ${ }^{24}$ According to this report, we examined our hypothesis in the above experimental setting. Glucosamine but not $\mathrm{N}$-acetylglucosamine strongly prevented the generation of the RP S19 dimer (data not shown). To further examine the participation of platelet membrane phosphatidylserine, we used annexin $V$ instead of glucosamine, because annexin $V$ is thought to bind phosphatidylserine in a specific manner. As shown in Figure 7, the simultaneous presence of annexin $\mathrm{V}$ prevented the generation of the monocyte chemotactic factor in a dose-dependent manner, and at $10^{-7} \mathrm{M}$ annexin $\mathrm{V}$, the chemotactic factor generation became negligible.

To confirm the participation of membrane phosphatidylserine, we substituted the platelets by lecithin-based liposomes either bearing phosphatidylserine at the phospholipid composition ratios of $30 \%$ or $10 \%$, or with no phosphatidylserine, and induced plasma clotting with CA-1. From the average weight of a platelet $(12.8 \mathrm{pg})$, platelet density in platelet-rich plasma $\left(4 \times 10^{8}\right.$ cells $\left./ \mathrm{ml}\right)$, the total lipid weight (4 pg/100 pg platelets), the ratio of total phospholipid to the total lipid in the platelet (76\%) and the ratio of phosphatidylserine in the total phospholipid (10\%), the concentration of platelet phosphatidylserine in platelet-rich plasma was calculated to be 15.6 $\mu \mathrm{g} / \mathrm{ml}$. We mixed liposomes with the platelet-poor plasma at the concentration of $78 \mu \mathrm{g} / \mathrm{ml}$. Thus, either 7.8 or 23.4 $\mu \mathrm{g} / \mathrm{ml}$ of phosphatidylserine was present in the cases of 10 and 30\% phosphatidylserine-containing liposomes, respectively. Because the liposome naturally shed some amounts of lysolecithin, which has monocyte chemotactic capacity, ${ }^{25,26}$ we tried to remove free lysolecithin by treatment of the plasma-derived sera with ether, and then measured the monocyte chemotactic capacities of the samples. The results are shown in Figure 8. The higher monocyte chemotactic capacity was generated depend-

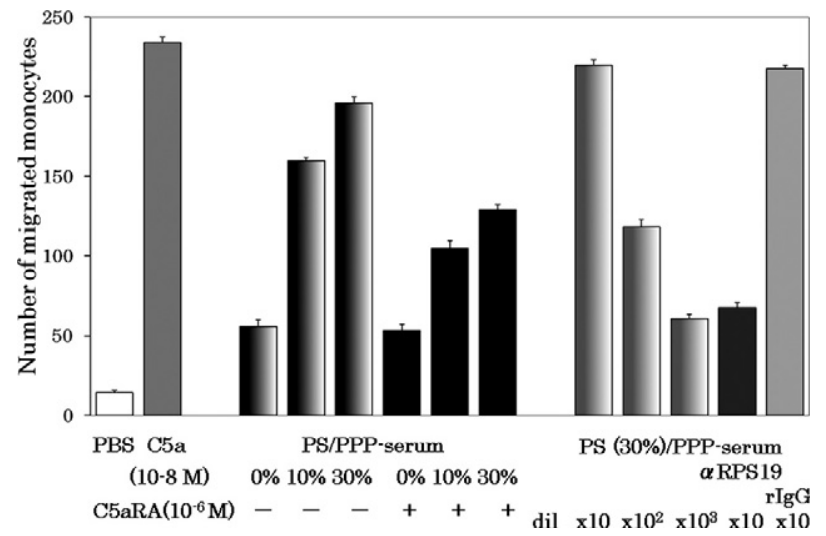

Figure 8. Substitution of platelets by phosphatidylserine-containing liposomes in the generation of monocyte-attracting factor during plasma coagulation. Aliquots of PPP were incubated with lecithin-based liposomes containing 30,10 , or $0 \%$ phosphatidylserine (PS) for 90 minutes at $37^{\circ} \mathrm{C}$. After the complement deactivation, the supernatants of the samples which correspond to the sera (PS/PPP-serum) were subjected to the monocyte chemotactic assay under the absence $(-)$ or presence $(+)$ of the $\mathrm{C} 5$ a receptor antagonist (C5aRA) at $10^{-6} \mathrm{M}$. The PS(30\%)/PPP-serum was then treated with either anti-RP S19 rabbit IgG-beads ( $\alpha$ RPS19 Ab) or with normal rabbit IgG beads (rIgG) in batchwise twice, and the monocyte chemotactic capacity remained was measured. Positive and negative controls for the chemotaxis assay were $10^{-8} \mathrm{M} \mathrm{C} 5 \mathrm{a}$ and PBS, respectively.

ing on the higher composition ratio of phosphatidylserine, and the chemotactic activity was sensitive to the C5a receptor antagonist, suggesting the dimer of RP S19-like molecule was generated on the phosphatidylserine-containing liposome. This was confirmed by the experiments using the anti-RP S19 antibody-beads. As also shown in Figure 8 , the monocyte chemotactic capacity above the base-line activity generated with the $30 \%$ phosphatidylserine-containing liposome was adsorbed by the anti-RP S19 antibody beads but not by the control IgG beads.

\section{Replacement of the RP S19-Like Molecule in Plasma with Recombinant RP S19 and Failure with Mutants of RP S19}

The above experiments indicated the presence of a molecule immunologically indistinguishable from RP S19 in plasma. To examine this in another way, we tried to replace the RP S19-like molecule in plasma with a recombinant RP S19. In this experiment, we first removed the RP S19-like molecule by the pretreatment of platelet-poor plasma twice with the anti-RP S19 antibody beads, and then reconstituted it with a recombinant wild-type RP S19 at final concentrations of $1 \mu \mathrm{g} / \mathrm{ml}$ and $10 \mu \mathrm{g} / \mathrm{ml}$. We arbitrarily determined the concentration of RP S19 reconstitution concerning the monocyte chemotactic capacity of the plasma-derived serum to be several hundred ng/ $\mathrm{ml}$. After adding the washed platelets, we prepared the plasma-derived serum with CA-1 protease and measured the monocyte chemotactic capacity that appeared.

To generate the functional RP S19 dimer possessing monocyte chemotactic capacity, the proper intermolecular isopeptide bond formation between Gln137 and Lys122 is needed. The functional dimer is not produced from a Gln137Asn-RP S19 mutant (named M12). ${ }^{3}$ There- 


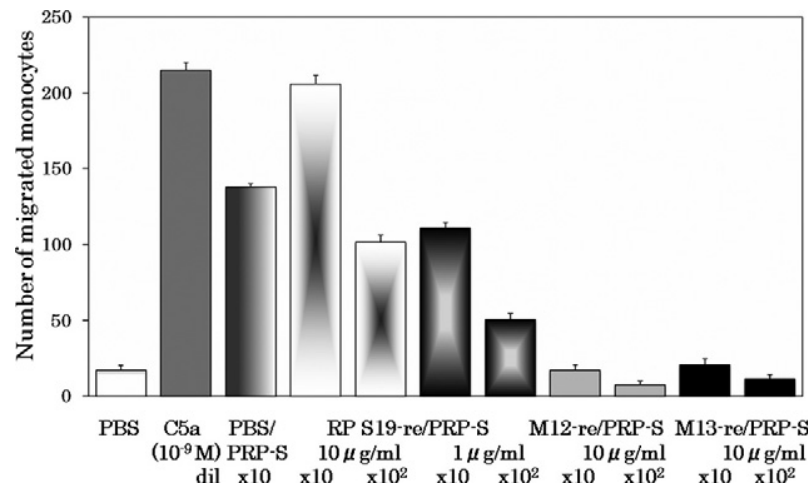

Figure 9. Replacement of RP S19-like molecule in plasma with recombinant RP S19 and with mutants of RP S19. PPP was pretreated with the anti-RP S19 rabbit IgG beads as Figure 4. Aliquots of it were reconstituted with a recombinant wild-type RP S19 (RPS19-re/) at concentrations of 1 or of 10 $\mu \mathrm{g} / \mathrm{ml}$, or with one of the RP S19 mutants, Gln137Asn-RP S19 (M12-re/), or Lys23, 24, 27, 29Ala-RP S19 (M13-re/) at a concentration of $10 \mu \mathrm{g} / \mathrm{ml}$ and were further added with platelets to reconstitute the PRP. After being coagulated with CA-1, the sera derived from the reconstituted PRP aliquots were heat treated (PRP-S) and subjected to the monocyte chemotaxis assay at serial log-scale dilutions. Positive control for the experiment was a serum derived from normal PRP compensated for the volume change with PBS (PBS/PRP-S). Positive and negative controls for the chemotaxis assay were $10^{-9} \mathrm{M} \mathrm{C} 5 \mathrm{a}$ and PBS, respectively.

fore, as a negative control, we reconstituted the immunodepleted plasma with Gln137Asn-RP S19 at the concentration of $10 \mu \mathrm{g} / \mathrm{m}$.

The basic cluster composed of Lys23, Lys24, Lys27, and Lys29 was revealed to be essential for the binding of the RP S19 molecule to heparin. ${ }^{3}$ Because phosphatidylserine on the activated platelets would work as negatively charged scaffold for the factor XIIla-catalyzed monocyte chemotactic factor generation during clotting of the platelet-rich plasma as described above, the basic cluster on the RP S19 molecule should be essential if RP S19 could replace the RP S19-like molecule. We therefore used a Lys23, 24, 27, 29Ala-RP S19 mutant (named M13) to reconstitute the immunodepleted plasma as another control at the concentration of $10 \mu \mathrm{g} / \mathrm{ml}$. As shown in Figure 9, monocyte chemotactic activity emerged when the plasma RP S19-like molecule was replaced by the wildtype RP S19 in the case of platelet-rich plasma-derived serum. In contrast, the monocyte chemotactic activity emerged only at greatly reduced extents in the sera when the plasma was reconstituted with GIn137Asn-RP S19 (M12) or with Lys23, 24, 27, 29Ala-RP S19 (M13). These experiments indicated that the factor XIlla-catalyzed isopeptide bond formation between GIn137 and a lysine residue, probably Lys122, were essential to gain the monocyte attracting capacity, and that the submolecular region of RP S19 bound to the surface phosphatidylserine molecules of the activated platelets was the basic cluster from Lys23 to Lys 29.

\section{Discussion}

In the present article, we report the presence of a plasma protein indistinguishable from RP S19 that is a component of the small subunit of the ribosome. In terms of the molecular size, the immunological reactivity, and the presence of the monocyte-attracting and neutrophilimmobilizing dual function after being cross-linked by a transglutaminase-catalyzed reaction, the plasma protein was indistinguishable from RP S19. The series of replacement experiments of the RP S19-like molecule in plasma with recombinant wild-type and mutant types (M12 and M13) of RP S19 also support the identity between the RP S19-like molecule and RP S19. However, the direct evidence to identify that the monocyte chemotactic RP S19like molecule is indeed the homodimer of it is still lacking. In the present article, we show Western blotting data generated using rabbit IgG raised to the synthetic Cterminal fragment of RP S19. There was no protein having a homologous sequence to this portion of the protein in our database search. We ran an immunodepletion of the RP S19-like molecule in plasma using anti-RP S19 whole protein rabbit IgG beads. We think that the molecule must be RP S19 itself from the evidence shown in the present report. An alternative interpretation was that the RP S19-like molecule might be RP S19 derived from platelet ribosomes or newly synthesized by platelets on thrombin activation. The band immunoreactive to the anti-RP S19 antibodies was observed in the platelet-poor plasma, and when the platelet-poor plasma was pretreated with anti-RP S19 antibody beads, the monocyte attracting capacity did not appear even though it was reconstituted with washed platelets and then coagulated. Hence, we conclude the RP S19-like molecule is present in plasma. However, the fact that a ribosomal protein is present in plasma seems unlikely. Therefore, we named the plasma protein as an RP S19-like molecule in the present article. We think that the final conclusion will be made after we have more data on the amino acid sequence or more accurate molecular size as analyzed by mass spectrum of the plasma protein after its purification.

At this moment we have no method to measure the concentration of the RP S19-like molecule in plasma. We have a plan to establish an enzyme-linked immunosorbent assay for RP S19 that would measure the concentration of the plasma RP S19-like molecule. Judging from the monocyte chemotactic capacities of natural serum and the serum derived from plasma replaced with the recombinant RP S19, the plasma concentration of the RP S19-like molecule is assumed to be several hundred nanograms per milliliter.

The experiments using factor XIII-deficient plasma with or without reconstitution by purified factor XIII demonstrated an essential role of factor XIII in the conversion of the RP S19-like molecule to its active form. The inhibition of the conversion with p-chloromercuriphenylsulfonic acid indicated the requirement of the catalytically active transglutaminase, ie, factor XIIla, because among the clotting enzymes, only factor XIIla is a thiol enzyme sensitive to p-chloromercuriphenylsulfonic acid. The factor XIIla crosslinks fibrin and fibronectin molecules as the major substrates to form their stable polymers. The RP S19-like molecule is converted to an active chemotactic factor by factor XIIla. This indicates that the active form conversion should be the cross-linking dimerization of the RP S19-like molecule due to isopeptide bond formation, as in the case of RP S19 dimerization in apoptotic cells. Judging from the slow 
generation of the active form (tens of minutes), we assume that the dimerization of the RP S19-like molecule occurs subsequent to fibrin cross-linkage.

The present study has demonstrated the role of phosphatidylserine in the outer leaflet of activated platelet plasma membranes on the conversion of the RP S19-like molecule to the active chemotactic factor. The activation was blocked by the simultaneous presence of phosphatidylserine-binding molecules (Figure 7), and the activated platelets could be substituted by phosphatidylserinecontaining liposomes but not by phosphatidylserine-free liposomes (Figure 8). Phosphatidylserine is thought to work as a scaffold in the factor XIIla-catalyzed dimerization of the RP S19-like molecule. The phosphatidylserine is constitutively located in the inner leaflet of the plasma membrane, and its externalization is caused by a phospholipid scramblase on the activation of platelets. ${ }^{23}$

The present findings will help us understand how RP S19 is dimerized in apoptosis-initiated cells by the cytoplasmic transglutaminases, such as type 2 transglutaminase, on the activation of the enzyme by the increase in cytoplasmic $\mathrm{Ca}^{2+} .{ }^{5}$ Getting a hint from the current study, we should assume that phosphatidylserine would be still present in the inner leaflet of plasma membrane at this period in the apoptotic process and would work as the scaffold for the RP S19 dimerization reaction. Furthermore, this study has brought us an idea for the extracellular secretion mechanism of the RP S19 dimer from the apoptotic cells: the translocation of phosphatidylserine from the inner leaflet to outer leaflet of plasma membrane occurring at the late phase of the apoptotic process by a phospholipid scramblase ${ }^{23}$ could play a role in the extracellular RP S19 dimer liberation.

The RP S19-like molecule in plasma was apparently converted to a dimer possessing a major monocyte attracting capacity in serum. For the biological role of the RP S19-like molecule dimer generated during blood coagulation, we have a working hypothesis that the dimer recruits monocytes/macrophages to the thrombus, blood coagulum, or hematoma to resolve these lesions by macrophage-dependent cellular fibrinolysis. Indeed, there are reports that thrombi experimentally formed in the blood stream were rapidly covered by monocytes ${ }^{27,28}$ that had been recruited from the blood stream. ${ }^{29}$ We recently found that the RP S19like molecule was also present in guinea pig plasma and converted to a dimer, gaining the monocyte-attracting capacity as in the case of humans. To experimentally examine our hypothesis of the biological role of the dimer, we recently established a coagulum resorption model in the guinea pig peritoneum. We have obtained experimental results that support our hypothesis and will report these results elsewhere.

\section{References}

1. Yamamoto T: Molecular mechanism of monocyte predominant infiltration in chronic inflammation: mediation by a novel monocyte chemotactic factor, S19 ribosomal protein dimer. Pathol Internat 2000, 50:863-871

2. Yamamoto T: Roles of the ribosomal protein S19 dimer and the C5a receptor in pathophysiological functions of phagocytic leukocytes. Pathol Internat 2007, 57:1-11
3. Nishiura H, Tanase S, Shibuya Y, Nishimura T, Yamamoto T: Determination of the cross-linked residues in homo-dimerization of S19 ribosomal protein concomitant with exhibition of monocyte chemotactic activity. Lab Invest 1999, 79:915-923

4. Horino K, Nishiura H, Ohsako T, Shibuya Y, Hiraoka T, Kitamura N Yamamoto T: A monocyte chemotactic factor. S19 ribosomal protein dimer, in phagocytic clearance of apoptotic cells. Lab Invest 1998, 78:603-617

5. Nishimura T, Horino K, Nishiura H, Shibuya Y, Hiraoka T, Tanase S, Yamamoto T: Apoptotic cells of an epithelial cell line, AsPC-1, release monocyte chemotactic S19 ribosomal protein dimer. J Biochem 2001, 129:445-454

6. Nishiura H, Tanase S, Shibuya Y, Futa N, Sakamoto T, Higginbottom A, Monk P, Zwirner J, Yamamoto T: S19 ribosomal protein dimer augments metal-induced apoptosis in a mouse fibroblastic cell line by ligation of the C5a receptor. J Cell Biochem 2005, 94:540-553

7. Nishiura H, Shibuya $Y$, Matsubara S, Tanase S, Kambara T, Yamamoto $\mathrm{T}$ : Monocyte chemotactic factor in rheumatoid arthritis synovial tissue: probably a cross-linked derivative of S19 ribosomal protein. J Biol Chem 1996, 271:878-882

8. Shi L, Tsurusaki S, Futa N, Sakamoto T, Matsuda T, Nishino N, Kunitomo R, Kawasuji M, Tokita K, Yamamoto T: Monocyte chemotactic S19 ribosomal protein dimer in atherosclerotic vascular lesion. Virchow Archiv 2005, 447:747-755

9. Nishiura H, Shibuya Y, Yamamoto T: S19 ribosomal protein cross-linked dimer causes monocyte-predominant infiltration by means of molecular mimicry to complement C5a. Lab Invest 1998, 78:1615-1623

10. Shrestha A, Horino K, Nishiura H, Yamamoto T: Acquired immune response as a consequence of the macrophage-dependent apoptotic cell clearance and role of the monocyte chemotactic S19 ribosomal protein dimer in this connection. Lab Invest 1999, 79:1629-1642

11. Oda Y, Tokita K, Ota Y, Li Y, Taniguchi K, Nishino N, Takagi K, Yamamoto T, Nishiura $\mathrm{H}$ : Agonistic and antagonistic effects of C5achimera bearing S19 ribosomal protein tail portion on the C5a receptor of monocytes and neutrophils, respectively. J Biochem 2008, 144:371-381

12. Kukita I, Yamamoto T, Kawaguchi T, Kambara T: Fifth component of complement (C5)-derived high molecular weight macrophage chemotactic factor in normal guinea pig serum. Inflammation 1987, 11:459-479

13. Okamoto M, Yamamoto T, Matsubara S, Kukita I, Takeya M, Miyauchi Y, Kambara T: Factor XIII-dependent generation of 5th complement component(C5)-derived monocyte chemotactic factor coinciding with plasma clotting. Biochim Biophys Acta 1992, 1138:53-61

14. Yamada D, Sekiya F, Morita T: Isolation and characterization of carinactivase, a novel prothrombin activator in Echis carinatus venom with a unique catalytic mechanism. J Biol Chem 1996, 271:5200-5207

15. Konteatis ZD, Siciliano SJ, Van-Riper G, Molineaux CJ, Pandya S, Fischer P, Rosen H, Mumford RA, Springer MS: Development of C5a receptor antagonists: differential loss of functional responses. J Immunol 1994, 153:4200-4205

16. Shibuya Y, Shiokawa M, Nishiura H, Nishimura T, Nishino N, Okabe H, Takagi K, Yamamoto T: Identification of receptor binding sites of monocyte chemotactic S19 ribosomal protein dimer. Am J Pathol 2001, 159:2293-2301

17. Fernandez HN, Henson PM, Otani A, Hugli TE: Primary structural analysis of the polypeptide portion of human $\mathrm{C} 5 \mathrm{a}$ anaphylatoxin: polypeptide sequence determination and assignment of the oligosaccharide attachment site in C5a. J Immunol 1978, 120:109-117

18. Matsubara S, Yamamoto T, Tsuruta T, Takagi K, Kambara T: Complement C4-derived monocyte-directed chemotaxis-inhibitory factor: a molecular mechanism to cause polymorphonuclear leukocyte-predominant infiltration in rheumatoid arthritis synovial cavities. Am J Pathol 1991, 138:1279-1291

19. Falk W, Goldwin RH, Leonard EJ: A 48 well micro-chemotaxis assembly for rapid and accurate measurement of leukocyte migration. J Immunol Methods 1980, 33:239-247

20. Cornwell DG, Kruger FA: Molecular complexes in the isolation and characterization of plasma lipoproteins. J Lipid Res 1961, 2: $110-134$

21. Dittrich M, Birschmann I, Pfrang J, Herterich S, Smolenski A, Walter U, Dandekar T: Analysis of SAGE data in human platelets: features of the transcriptome in an anucleate cell. Thromb Haemost 2006, 95:643-651 
22. Zimmerman GA, Weyrich AS: Signal-dependent protein synthesis by activated platelets: new pathways to altered phenotype and function. Arterioscler Thromb Vasc Biol 2008, 28:s17-s24

23. Sahu SK, Gummadi SN, Manoj N, Aradhyam GK: Phospholipid scramblase: an overview. Archiv Biochem Biophys 2007, 462:103-114

24. Savill JS, Henson PM, Haslett C: Phagocytosis of aged human neutrophils by macrophages is mediated by a novel "chargesensitive" recognition mechanism. J Clin Invest 1989, 84: 1518-1527

25. Quinn MT, Parthasarathy S, Steinberg D: Lysophosphatidylcholine: a chemotactic factor for human monocytes and its potential role in atherogenesis. Proc Natl Acad Sci USA 1988, 85:2805-2809

26. Jing Q, Xin SM, Zang WB, Wang P, Qin YW, Pei G: Lysophosphati- dylcholine activates p38 and p42/44 mitogen-activated protein kinases in monocytic THP-1 cells, but only p38 activation is involved in its stimulated chemotaxis. Circ Res 2000, 87:52-59

27. Davies MJ, Ballantine SJ, Robertson WB, Woolf N: The ultrastructure of organizing experimental mural thrombi in the pig aorta. $J$ Pathol 1975, 117:75-81

28. Feigl W, Susani M, Ulrich W, Matejka M, Losert U, Sinzinger $H$ Organisation of experimental thrombosis by blood cells: evidence of the transformation of mononuclear cells into myofibroblasts and endothelial cells Virchows Arch 1985, 406:133-148

29. McGuinness CL, Humphries J, Waltham M, Burnand K, Collins GM Smith A: Recruitment of labeled monocytes by experimental venous thrombi. Thromb Haemost 2001, 85:1018-1024 